\title{
Medication Therapy of High-Dose Methotrexate: An Evidence-Based Practice Guideline Division of Therapeutic Drug Monitoring, Chinese Pharmacological Society
}

Song Zaiwei ${ }^{1}$, Yang $\mathrm{Hu}^{1}$, Shuang Liu ${ }^{1}$, Guanru Wang ${ }^{1}$, Suo-Di Zhai ${ }^{1}$, Xianglin Zhang ${ }^{2}$, Youping $\mathrm{Li}^{3}$, Guanhua Du ${ }^{4}$, Yuankai Shi ${ }^{4}$, Yaolong Chen ${ }^{5}$, Mei Dong ${ }^{6}$, Guo Rui-chen ${ }^{7}$, Wei $\mathrm{Guo}^{8}$, Hongbing Huang ${ }^{9}$, Xiaojun Huang ${ }^{8}$, Hongmei Jing ${ }^{1}$, Xiaoyan $\mathrm{Ke}^{1}$, Guohui Li ${ }^{4}$, Liyan $\mathrm{Miao}^{10}$, Xiaohui Niu ${ }^{11}$, Feng Qiu ${ }^{12}$, Jingnan Shen ${ }^{13}$, Jingyan Tang ${ }^{14}$, Tian-You Wang ${ }^{15}$, Xiaoling Wang ${ }^{15}$, Zhuo Wang ${ }^{16}$, Jiuhong Wu ${ }^{17}$, Si-Yan Zhan ${ }^{18}$, Bi-kui Zhang ${ }^{19}$, Lingli Zhang $^{20}$, Yanhua Zhang ${ }^{21}$, Wei Zhang ${ }^{22}$, Li Zhao ${ }^{23}$, Li-bo Zhao ${ }^{15}$, Jiancun Zhen ${ }^{22}$, Huyong Zheng ${ }^{15}$, Zhu Zhu ${ }^{24}$, Dan Jiang ${ }^{1}$, Zhencheng Huang ${ }^{1}$, Zhiyuan Tan ${ }^{1}$, Qiaonan Lin ${ }^{1}$, and Rongsheng Zhao ${ }^{1}$

${ }^{1}$ Peking University Third Hospital

${ }^{2}$ China-Japan Friendship Hospital

${ }^{3}$ Sichuan University West China Hospital

${ }^{4}$ Chinese Academy of Medical Sciences and Peking Union Medical College

${ }^{5}$ Lanzhou University School of Basic Medical Sciences

${ }^{6}$ Harbin Medical University Third Hospital

${ }^{7}$ Shandong University Qilu Hospital

${ }^{8}$ Peking University People's Hospital

${ }^{9}$ Sun Yat-sen University Cancer Center

${ }^{10}$ First Affiliated Hospital of Soochow University

${ }^{11}$ Beijing Jishuitan Hospital

${ }^{12}$ The First Affiliated Hospital of Chongqing Medical University

${ }^{13}$ Sun Yat-sen University First Affiliated Hospital

${ }^{14}$ Shanghai Childrens Medical Center Affiliated to Shanghai Jiaotong University School of Medicine

${ }^{15}$ Beijing Children's Hospital Capital Medical University

${ }^{16}$ Changhai Hospital

${ }^{17}$ The 306th Hospital of People's Liberation Army

${ }^{18}$ Peking University School of Public Health

${ }^{19}$ Second Xiangya Hospital

${ }^{20}$ Sichuan University West China Second University Hospital

${ }^{21}$ Peking University Cancer Hospital

${ }^{22}$ Jishuitan Hospital and Fourth Medical College of Peking University

${ }^{23}$ Shengjing hospital of China medical university

${ }^{24}$ Peking Union Medical College Hospital,Chinese Academy of Medical Sciences,

June 3, 2021 


\begin{abstract}
Objectives: A lot of medication risks related to high-dose methotrexate (HDMTX) therapy still remain to be identified and standardized. This study aims to establish an evidence-based practice guideline for individualized medication of HDMTX. Methods: The practice guideline was launched by the Division of Therapeutic Drug Monitoring, Chinese Pharmacological Society. The guideline was developed following the WHO handbook for guideline development and the methodology of evidence-based medicine (EBM). The guideline was initially registered in the International Practice Guidelines Registry Platform (IPGRP2017CN021). Systematic reviews were conducted to synthesis available evidence. A multicenter cross-sectional study was conducted by questionnaires to evaluate patients' perception and willingness on individualized medication of HDMTX. The Grading of Recommendations Assessment, Development, and Evaluation (GRADE) approach was used to rate the quality of evidence and to grade the strength of recommendations. Results: Multidisciplinary working groups were included in this guideline, including clinicians, pharmacists, methodologists, pharmacologists and pharmacoeconomic specialists. A total of 124 patients were involved to integrate patient values and preferences. Finally, the guideline presents 28 recommendations, regarding evaluation prior to medication (renal function, liver function, pleural effusion, comedications, genetic testing), pre-treatment and routine dosing regimen, therapeutic drug monitoring (necessity, method, timing, target concentration), leucovorin rescue (initial timing, dosage regimen and optimization), management of toxicities. Of them, 12 are strong recommendations. Conclusions: We developed an evidence-based practice guideline with respect to HDMTX medication using a rigorous and multidisciplinary approach. This guideline provides comprehensive and practical recommendations involving the whole process of HDMTX medication to health care providers.
\end{abstract}

Title : Medication Therapy of High-Dose Methotrexate: An Evidence-Based Practice Guideline Division of Therapeutic Drug Monitoring, Chinese Pharmacological Society

\title{
Author names and affiliations :
}

Zaiwei Song ${ }^{1,2,3}$, Yang $\mathrm{Hu}^{1,2,3}$, Shuang Liu ${ }^{1,2,3}$, Guanru Wang ${ }^{1,2,3}$, Suodi Zhai ${ }^{1,2,3}$, Xianglin Zhang ${ }^{4}$, Youping $\mathrm{Li}^{5}$, Guanhua $\mathrm{Du}^{6}$, Yuankai Shi ${ }^{7}$, Yaolong Chen ${ }^{8}$, Mei Dong ${ }^{9}$, Ruichen Guo ${ }^{10}$, Wei Guo ${ }^{11}$, Hongbing Huang ${ }^{12}$, Xiaojun Huang ${ }^{13}$, Hongmei Jing ${ }^{14}$, Xiaoyan $\mathrm{Ke}^{14}$, Guohui $\mathrm{Li}^{15}$, Liyan Miao ${ }^{16}$, Xiaohui Niu ${ }^{17}$, Feng $\mathrm{Qiu}^{18}$, Jingnan Shen ${ }^{19}$, Jingyan Tang ${ }^{20}$, Tianyou Wang ${ }^{21}$, Xiaoling Wang ${ }^{22}$, Zhuo Wang ${ }^{23}$, Jiuhong $\mathrm{Wu}^{24}$, Siyan Zhan ${ }^{25}$, 26, Bikui Zhang ${ }^{27}$, Lingli Zhang ${ }^{28}$, Yanhua Zhang ${ }^{29}$, Wei Zhang ${ }^{30}$, Limei Zhao ${ }^{31}$, Libo Zhao ${ }^{22}$, Jiancun Zhen ${ }^{30}$, Huyong Zheng ${ }^{32}$, Zhu Zhu ${ }^{33}$, Dan Jiang ${ }^{1,2,3}$, Zhencheng Huang ${ }^{1,2,3}$, Zhiyuan Tan ${ }^{1,2,3}$, Qiaonan $\operatorname{Lin}^{1,2,3}$, Rongsheng Zhao ${ }^{1,2,3 *}$

1. Department of Pharmacy, Peking University Third Hospital, Beijing 100191, China

2. Institute for drug evaluation, Peking University Health Science Center, Beijing 100191, China

3. Therapeutic Drug Monitoring and Clinical Toxicology Center, Peking University, Beijing 100191, China

4. Department of Pharmacy, China-Japan Friendship Hospital, Beijing 100029, China

5. Chinese Evidence-Based Medicine Center, West China Hospital, Sichuan University, Chengdu 610041, China

6. Institute of Materia Medica, Chinese Academy of Medical Sciences \& Peking Union Medical College, Beijing 100050, China

7. Department of Medical Oncology, Cancer Hospital, Chinese Academy of Medical Sciences \& Peking Union Medical College, National Cancer Center/National Clinical Research Center for Cancer, Beijing 100021, China

8. Evidence-Based Medicine Center, School of Basic Medical Sciences, Lanzhou University, Lanzhou 730000, China

9. Department of Pharmacy, Harbin Medical University Cancer Hospital, Harbin 150040, China

10. Institute of Clinical Pharmacology, Qilu Hospital of Shandong University, Jinan 250012, China 
11. Musculoskeletal Tumor Center, Peking University People's Hospital, Beijing 100044, China

12. Department of Pharmacy, Collaborative Innovation Center for Cancer Medicine, Sun Yat-sen University Cancer Center, Guangzhou 510060, China

13. Peking University Institute of Hematology, Peking University People's Hospital, National Clinical Research Center for Hematologic Disease, Beijing 100044, China

14. Department of Hematology, Peking University Third Hospital, Beijing 100191, China

15. Department of Pharmacy, Cancer Hospital, Chinese Academy of Medical Sciences \& Peking Union Medical College, National Cancer Center/National Clinical Research Center for Cancer, Beijing 100021, China

16. Department of Clinical Pharmacology, First Affiliated Hospital of Soochow University, Suzhou 215006, China

17. Department of Orthopedic Oncology, Beijing Jishuitan Hospital and Fourth Medical College of Peking University, Beijing 100035, China

18. Department of Pharmacy, The first Affiliated Hospital of Chongqing Medical University, Chongqing 400042, China

19. Department of Musculoskeletal Oncology, The First Affiliated Hospital of Sun Yat-Sen University, Guangzhou 510080, China

20. Department of Hematology/Oncology, Shanghai Children's Medical Center, Shanghai Jiao Tong University School of Medicine, Key Lab of Pediatric Hematology \& Oncology of China Ministry of Health, Shanghai 200127, China

21. Hematology Oncology Center, Beijing Children's Hospital, Capital Medical University, National Center for Children's Health, Beijing, 100045, China

22. Department of Pharmacy, Beijing Children's Hospital, Capital Medical University, Beijing 100045, China

23. Department of Pharmacy, Shanghai Changhai Hospital, Second Military Medical University, Shanghai 200433, China

24. Pharmacy Department, The 306th Hospital of PLA, Beijing 100101, China

25. Department of Epidemiology and Biostatistics, School of Public Health, Peking University, Beijing 100083, China

26. Research Center of Clinical Epidemiology, Peking University Third Hospital, Beijing 100191, China

27. Department of Pharmacy, The Second Xiangya Hospital of Central South University, Changsha 410011, China

28. Department of Pharmacy/Evidence-Based Pharmacy Centre, West China Second University Hospital, Sichuan University, Chengdu 610041, China

29. Department of Pharmacy, Peking University Cancer Hospital and Institute, Key Laboratory of Carcinogenesis and Translational Research (Ministry of Education), Beijing 100142, China

30. Department of Pharmacy, Jishuitan Hospital and Fourth Medical College of Peking University, Beijing 100035, China

31. Department of Pharmacy, Shengjing Hospital of China Medical University, Shenyang 110004, China

32. Hematology Oncology Center, Beijing Children's Hospital, Capital Medical University, National Center for Children's Health, Beijing 100045, China 
33.Department of Pharmacy, Peking Union Medical College Hospital, Chinese Academy of Medical Sciences \& Peking Union Medical College, Beijing 100730, China

\section{Contact information of all authors:}

Zaiwei Song (E-mail: songzw445@126.com)

Yang Hu (E-mail: huyang97@bjmu.edu.cn)

Shuang Liu (E-mail: liushuang_sss@126.com)

Guanru Wang (E-mail: Mangowang_China@pku.edu.cn)

Suodi Zhai (E-mail: zhaisuodi@163.com)

Xianglin Zhang (E-mail: zryhyyzxl@126.com)

Youping Li (E-mail: yzmylab@hotmail.com)

Guanhua Du (E-mail: dugh@imm.ac.cn)

Yuankai Shi (E-mail: syuankaipumc@126.com)

Yaolong Chen (E-mail: chenyaolong@lzu.edu.cn)

Mei Dong (E-mail: 13804567370@163.com)

Ruichen Guo (E-mail: grc7636@126.com)

Wei Guo (E-mail: bonetumor@163.com)

Hongbing Huang (E-mail: huanghb@sysucc.org.cn)

Xiaojun Huang (E-mail: xjhrm@medmail.com.cn)

Hongmei Jing (E-mail: hongmei_jing@163.com)

Xiaoyan Ke (E-mail: xykbysy@163.com)

Guohui Li (E-mail: lgh0603@126.com)

Liyan Miao (E-mail: miaolysuzhou@163.com)

Xiaohui Niu (E-mail: niuxiaohui@263.net)

Feng Qiu (E-mail: qiufeng.cn@outlook.com)

Jingnan Shen (E-mail: shenjingnan@126.com)

Jingyan Tang (E-mail: tangjingyan@scmc.com.cn)

Tianyou Wang (E-mail: wangtianyou@bch.com.cn)

Xiaoling Wang (E-mail: wangxiaoling@bch.com.cn)

Zhuo Wang (E-mail: wangzhuo088@163.com)

Jiuhong Wu (E-mail: jiuhongwu2015@163.com)

Siyan Zhan (E-mail: siyan-zhan@bjmu.edu.cn)

Bikui Zhang (E-mail: bikui_zh@126.com)

Lingli Zhang (E-mail: zhlingli@sina.com)

Yanhua Zhang (E-mail: zyh8812@163.com)

Wei Zhang (E-mail: jstzhangwei@126.com) 
Limei Zhao (E-mail: zhaolm@sj-hospital.org)

Libo Zhao (E-mail: lb.zhao@163.com)

Jiancun Zhen (E-mail: Zhenjiancun@163.com)

Huyong Zheng (E-mail: zhenghuyong@vip.sina.com)

Zhu Zhu (E-mail: zhuzhu@pumch.cn)

Dan Jiang (E-mail: jiangdan@bjmu.edu.cn)

Zhencheng Huang (E-mail: huangzhencheng@pku.edu.cn)

Zhiyuan Tan (E-mail: 13733832637@163.com)

Qiaonan Lin (E-mail: 18802452681@163.com)

Rongsheng Zhao (E-mail: zhaorongsheng@bjmu.edu.cn)

Corresponding author and present address : Rongsheng Zhao, Professor, Chief pharmacist, Doctor of Pharmacy, Department of Pharmacy, Peking University Third Hospital, 49 North Garden Rd., Haidian District, Beijing, China. E-mail: zhaorongsheng@bjmu.edu.cn; Telephone: 86-10-8226 5810; Fax: 86-10-6201 7700 .

\section{Conflicts of interest disclosure statements for all authors:}

All authors reported no potential conflicts of interest.

Statement of funding source:

This work was supported by the Division of Therapeutic Drug Monitoring, Chinese Pharmacological Society, and the National Natural Science Foundation of China (NSFC) (grant number 72074005).

Word count: 6366

Abstract word count : 248

Figure count: 2

Table count: 2

Hosted file

3-Manuscript-0517.docx available at https://authorea.com/users/417806/articles/524762medication-therapy-of-high-dose-methotrexate-an-evidence-based-practice-guidelinedivision-of-therapeutic-drug-monitoring-chinese-pharmacological-society 